\begin{tabular}{|c|c|}
\hline Title & $\begin{array}{l}\text { A PDF Est i mat i on- Based I ter at i ve M MO Si gnal } \\
\text { Det ect i on Wth Unknown I nt erf er ence }\end{array}$ \\
\hline Author(s) & $\begin{array}{l}\text { Vesel i novi c, Nenad; Nat sumbt o, Tadashi ; Junt ti, } \\
\text { Markku }\end{array}$ \\
\hline Citation & I EEE Communi cat i ons Let ter s, 8( 7): 422- 424 \\
\hline Issue Date & 2004-07 \\
\hline Type & Journal Arti cle \\
\hline Text version & publ i sher \\
\hline URL & ht t p: //hdl . handl e. net $/ 10119 / 4633$ \\
\hline Rights & 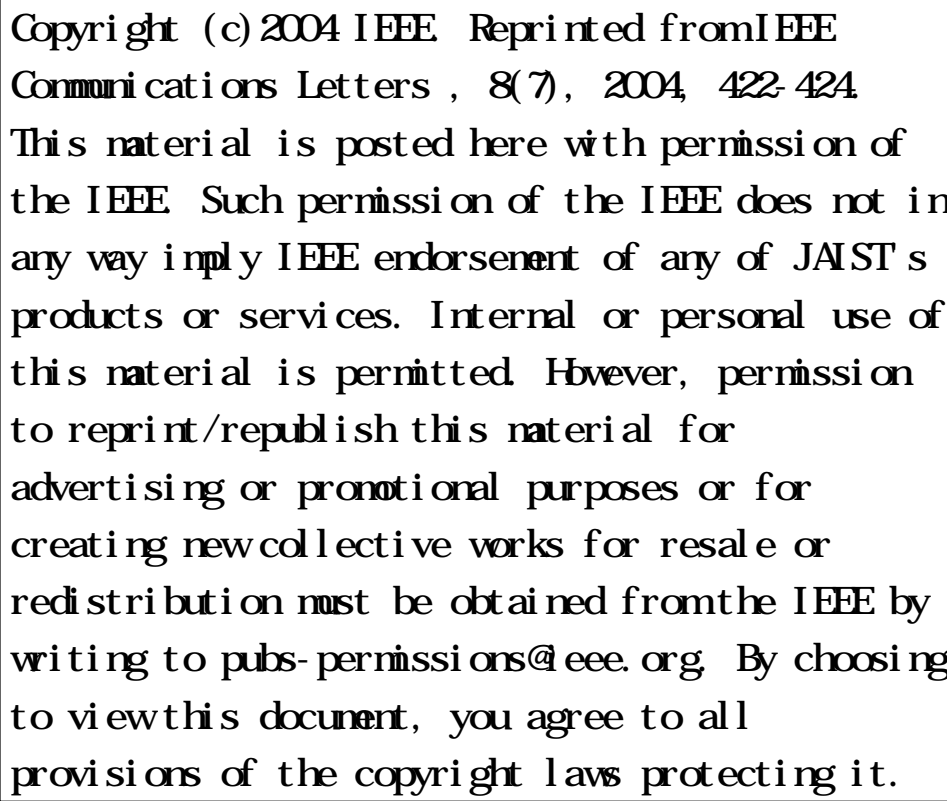 \\
\hline Descr & \\
\hline
\end{tabular}

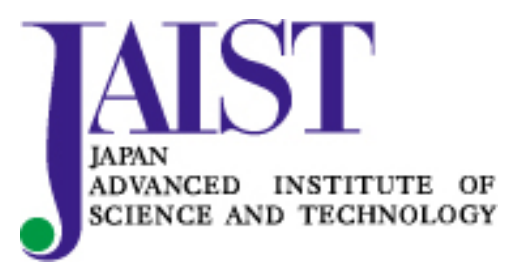




\title{
A PDF Estimation-Based Iterative MIMO Signal Detection With Unknown Interference
}

\author{
Nenad Veselinovic, Student Member, IEEE, Tadashi Matsumoto, Senior Member, IEEE, and \\ Markku Juntti, Senior Member, IEEE
}

\begin{abstract}
The equivalent diversity order of iterative minimum mean squared error (MMSE) multiuser detectors for multiple input multiple output (MIMO) channels is decreased if they aim at suppressing unknown cochannel interference (UCCI) while detecting multiple users' signals. In this letter, we propose a new MIMO signal detection scheme with the aim to preserve the detector's diversity order by estimating the probability density function of the UCCI plus background noise. It is shown that the proposed detector significantly outperforms the conventional detector based on UCCI's covariance matrix estimation.
\end{abstract}

Index Terms-Channel estimation, cochannel interference, equalization, multiple input multiple output (MIMO), minimum mean squared error (MMSE), probability density function (PDF) estimation, turbo multiuser detection.

\section{INTRODUCTION}

I TERATIVE low-complexity multiuser detection, equalization and decoding principle based on soft cancellation and minimum-mean squared-error filtering (SC/MMSE) [1] has attracted a considerable attention in recent years. It is a suboptimal solution to the prohibitively complex optimal joint multiuser detection, equalization and decoding problems.

The SC/MMSE equalizer is robust against unknown cochannel interference (UCCI) if the covariance matrix of the UCCI is properly estimated [2] or subspace estimation [3] is performed. To suppress UCCI, however, the both methods unavoidably consume the degrees-of-freedom (DOF) supported by the multiple antennas for MIMO signal detection, resulting in decreased diversity order [3]. In fact, the available DOF are used both for UCCI suppression and multipath combining. The loss tends to be more severe in lower memory length frequency selective channels, due to the lack of multipath diversity.

In this letter, we propose a new iterative receiver that can preserve the diversity gain by estimating the probability density function (PDF) of the UCCI plus noise. It is shown through simulations that the proposed receiver significantly outperforms the conventional detector of [2] in relatively low memory length channels.

The letter is organized as follows. Section II describes system model. Section III presents the proposed receiver. Sections IV and $\mathrm{V}$ present the simulation results and conclusions, respectively.

Manuscript received December 3, 2003. . The associate editor coordinating the review of this letter and approving it for publication was Dr. R. S. Blum. This work was supported by Nokia, Elektrobit, Finnish Air Forces, National Technology Agency of Finland (Tekes), Instrumentointi, Nokia Foundation, and Infotech Oulu Graduate School.

The authors are with the Centre for Wireless Communications, University of Oulu, Oulu, Finland (e-mail: nenad@ee.oulu.fi).

Digital Object Identifier 10.1109/LCOMM.2004.832731

\section{SYSTEM MODEL}

The system model is shown in Fig. 1. The notations follow closely those in [1]. Each of $N+N_{I}$ users encodes information sequence $c_{n}(i), n=1, \ldots, N+N_{I}, i=1, \ldots, B R$ with $R$ and $B$ being the code rate and frame length in symbols, respectively. Users indexed by $n=1, \ldots, N$ are desired users, and those indexed by $n=N+1, \ldots, N+N_{I}$ are assumed to be UCCI. The encoded sequences $b_{n}(k), k=1, \ldots, B$ are interleaved, and headed by the user-specific training sequences of length $T$. The entire frame is then BPSK modulated and transmitted through frequency selective channel with $L$ paths.

After coherent demodulation in the receiver, the signals from each of the $M$ receive antennas are sampled in time domain to capture the multipath components. The space-time representation of the received signal at time instant $k$ is given in the vector form by [1]

$$
\mathbf{y}(k)=\mathbf{H u}(k)+\mathbf{H}_{I} \mathbf{u}_{I}(k)+\mathbf{n}(k), \quad k=1, \ldots, T+B
$$

where $\mathbf{y}(k) \in \mathbb{C}^{L M}$ is the space-time sampled received signal vector and $\mathbf{H}$ is the channel matrix of the form

$$
\begin{aligned}
\mathbf{H} & =\left[\begin{array}{ccccc}
\mathbf{H}(0) & \ldots & \mathbf{H}(L-1) & \ldots & \mathbf{0} \\
\vdots & \ddots & & \ddots & \vdots \\
\mathbf{0} & \ldots & \mathbf{H}(0) & \ldots & \mathbf{H}(L-1)
\end{array}\right] \\
& \in \mathbb{C}^{L M \times N(2 L-1)}
\end{aligned}
$$

with $[\mathbf{H}(l)]_{m, n}$ being the $l$-th path complex gain between the $n$-th user and $m$ th receive antenna. $\mathbf{H}_{I} \in \mathbb{C}^{L M \times N_{I}(2 L-1)}$ is channel matrix of the UCCI defined similarly to $\mathbf{H}$, and $\mathbf{u}(k) \in \mathbb{C}^{N(2 L-1)}, \mathbf{u}_{I}(k) \in \mathbb{C}^{N_{I}(2 L-1)}$ and $\mathbf{n}(k) \in \mathbb{C}^{L M}$ are vectors containing desired users' signals, UCCI signals and additive white Gaussian noise (AWGN) with covariance $E\left\{\mathbf{n}(k) \mathbf{n}^{H}(k)\right\}=\sigma^{2} \mathbf{I}$, respectively [1].

\section{ITERATIVE RECEIVER WITH PDF ESTIMATION}

First iteration: In the first iteration the proposed receiver is exactly the same as conventional receiver from [2]. Let $\mathbf{x}(k)=$ $\mathbf{H}_{I} \mathbf{u}_{I}(k)+\mathbf{n}(k)$. Let $\tilde{\mathbf{u}}(k), k=1, \ldots, T$ denote the vector containing training sequences of $N$ desired users [1]. The samples $\tilde{\mathbf{u}}(k), k=1, \ldots, T$ are first fed to the channel estimator to obtain the estimate $\hat{\mathbf{H}}$ of $\mathbf{H}$. After that the samples $\hat{\mathbf{x}}(k)=$ $\mathbf{y}(k)-\hat{\mathbf{H}} \tilde{\mathbf{u}}(k), k=1, \ldots, T$ are used to estimate the covariance matrix of the UCCI-plus-noise using sample average given by

$$
\mathbf{R}_{\mathbf{x x}}=\mathbf{H}_{I} \mathbf{H}_{I}^{H}+\sigma^{2} \mathbf{I} \approx \frac{\sum_{k=1}^{T} \hat{\mathbf{x}}(k) \hat{\mathbf{x}}^{H}(k)}{T}=\hat{\mathbf{R}}_{\mathbf{x x}} .
$$




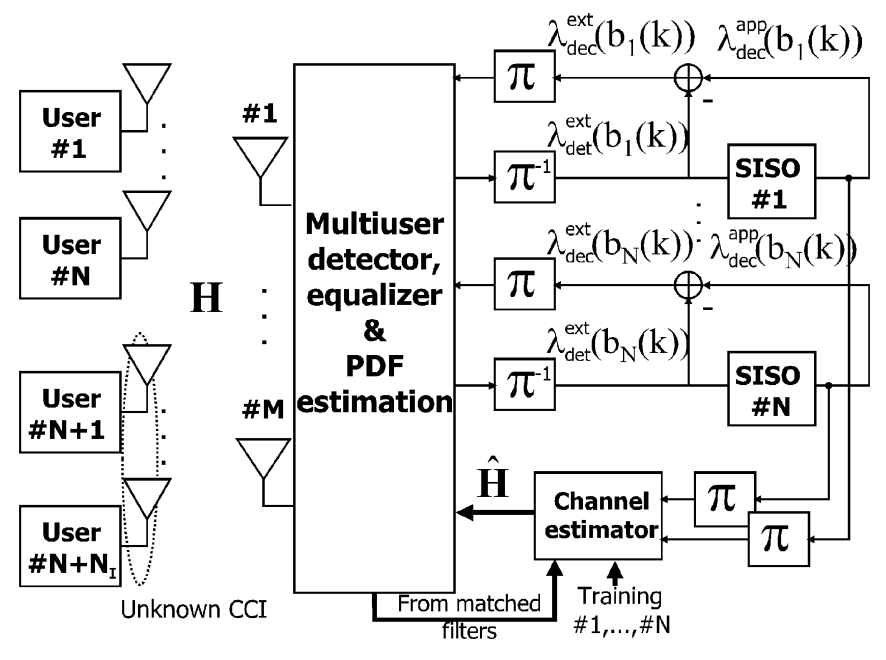

Fig. 1. Transmitter and iterative receiver block schemes.

Assume that the first user is of interest. In order to suppress the known and unknown CCI components as well as the ISI components of the first user, the signal $\mathbf{y}(k), k=T+1, \ldots, T+B$ is filtered with a linear MMSE filter [2] with weighting vector $\mathbf{w}_{1}(k)=\left[\hat{\mathbf{H}} \hat{\mathbf{H}}^{H}+\hat{\mathbf{R}}_{\mathbf{x x}}\right]^{-1} \hat{\mathbf{h}}_{1}$, where $\hat{\mathbf{h}}_{1}=\hat{\mathbf{H}} \mathbf{e}_{1}$ and $\mathbf{e}_{1}=\left[\begin{array}{lll}\mathbf{0}_{(L-1) N}^{T} & 1 & \mathbf{0}_{L N-1}^{T}\end{array}\right]^{T}$. The extrinsic information to be passed to the decoder is calculated as [1]

$\lambda_{\operatorname{det}}^{e x t}\left(b_{1}(k)\right)=\frac{4 \Re\left\{\mathbf{w}_{1}^{H}(k) \mathbf{y}(k)\right\}}{1-\mathbf{w}_{1}^{H}(k) \hat{\mathbf{h}}_{1}}, \quad k=T+1, \ldots, T+B$.

Subsequent iterations: Soft feedback obtained after decoding is concatenated with training sequences of desired users resulting in the enlarged set of training samples $\tilde{\mathbf{u}}(k), k=1, \ldots, T+B$. The channel is first re-estimated based on this enlarged set of samples [4]. After that, the estimates $\hat{\mathbf{x}}(k)=\mathbf{y}(k)-\hat{\mathbf{H}} \tilde{\mathbf{u}}(k)$, $k=1, \ldots, T+B$ are found. The soft cancellation is then performed, resulting in CCI- and ISI-free signal of the first user $\hat{\mathbf{y}}_{1}(k)=\mathbf{y}(k)-\hat{\mathbf{H}} \tilde{\mathbf{u}}_{1}(k), k=T+1, \ldots, T+B$, where $\tilde{\mathbf{u}}_{1}(k)=\tilde{\mathbf{u}}(k)-\tilde{\mathbf{u}}(k) \odot \mathbf{e}_{1}$, and $\odot$ denotes elementwise vector product. At this point the conventional receiver of [2] uses the enlarged set of samples $\hat{\mathbf{x}}(k), k=1, \ldots, T+B$ to make the estimate $\hat{\mathbf{R}}_{\mathbf{x x}}$, applies linear MMSE filter (see [2]) to the signal $\hat{\mathbf{y}}_{1}(k)$ and calculates extrinsic information as in (3), with $\hat{\mathbf{y}}_{1}(k)$ instead of $\mathbf{y}_{1}(k)$. Unlike that, the proposed receiver uses first $T$ samples $\hat{\mathbf{x}}(k), k=1, \ldots, T$ to make the estimate $\hat{p}_{\hat{\mathbf{x}}}$ of PDF $p_{\hat{\mathbf{x}}}$ of $\hat{\mathbf{x}}(k)$ and uses maximum likelihood (ML) detection to calculate extrinsic information

$\lambda_{\text {det }}^{e x t}\left(b_{1}(k)\right)=\log \frac{p\left(\hat{\mathbf{y}}_{1}(k) \mid b_{1}(k)=+1\right)}{p\left(\hat{\mathbf{y}}_{1}(k) \mid b_{1}(k)=-1\right)}=\log \frac{\hat{p}_{\hat{\mathbf{x}}}\left(\hat{\mathbf{y}}_{1}(k)-\hat{\mathbf{h}}_{1}\right)}{\hat{p}_{\hat{\mathbf{x}}}\left(\hat{\mathbf{y}}_{1}(k)+\hat{\mathbf{h}}_{1}\right)}$.

Assuming perfect soft-cancellation the true PDF $p_{\hat{\mathbf{x}}}$ is multimodal Gaussian, and it is given by

$$
p_{\hat{\mathbf{x}}}(\mathbf{a})=\frac{1}{2^{D}} \sum_{i=0}^{2^{D}-1} p_{G}\left(\mathbf{a}-\mathbf{t}_{i}\right)
$$

where $\mathbf{a}=\left[a_{1}, \ldots, a_{L M}\right]^{T} \in \mathbb{C}^{L M}, D=(2 L-1) N_{I}$, $p_{G}(\mathbf{a})=\left(1 /\left(2 \pi \sigma^{2}\right)^{L M}\right) e^{\left(-\mathbf{a}^{H} \mathbf{a} / 2 \sigma^{2}\right)}$ and vectors $\mathbf{t}_{i} \in \mathbb{C}^{L M}$ depend on the entries of $\mathbf{H}_{I}$. Instead of estimating parameters
$D$ and $\mathbf{t}_{i}$ and using them in (5) to obtain estimate $\hat{p}_{\hat{\mathbf{x}}}{ }^{1}$, we use kernel-smoothing [5], [6] that approximates $p_{\hat{\mathbf{x}}}$ directly with

$$
\hat{p}_{\hat{\mathbf{x}}}(\mathbf{a})=\frac{1}{T} \sum_{k=0}^{T-1} K_{1} \frac{\left(\frac{\mathbf{a}-\hat{\mathbf{x}}(k)}{\sigma_{0}}\right)}{\sigma_{0}^{2 L M}}
$$

where $K_{1}(\mathbf{a})=\left(1 /(2 \pi)^{L M}\right) e^{\left(-\mathbf{a}^{H} \mathbf{a} / 2\right)}$ is Gaussian kernel and $\sigma_{0}$ is the smoothing parameter [5] to be optimized. It will be shown in the sequel that this choice of kernel gives an asymptotically unbiased and consistent PDF estimator [6]. It will also be shown that receiver based on (6) does not require knowledge of $\mathbf{t}_{i}$ or $\mathbf{H}_{I}$, and that even no exact knowledge of $D$ is necessary for satisfactory performance in a variety of scenarios. To obtain the optimal value of $\sigma_{0}$ we minimize the mean-integrated-square-error (MISE) between $p_{\hat{\mathbf{x}}}$ and its estimate $\hat{p}_{\hat{\mathbf{x}}}$, where MISE is defined by [5]

$$
\operatorname{MISE}\left(\hat{p}_{\hat{\mathbf{x}}}\right) \approx \frac{\sigma_{0}^{4} \alpha^{2} \Gamma\left(p_{\hat{\mathbf{x}}}\right)}{4}+\frac{\int_{\mathbb{R}^{2 L M}} K_{1}^{2}(\mathbf{a}) d \mathbf{a}}{T \sigma_{0}^{2 L M}}
$$

where $d \mathbf{a}=d \Re a_{1} d \Im a_{1} \cdots d \Re a_{L M} d \Im a_{L M}, \alpha=\int_{\mathbb{R}^{2 L M}}$ $\left(\Re a_{1}\right)^{2} K_{1}(\mathbf{a}) d \mathbf{a}=1$

$$
\Gamma\left(p_{\hat{\mathbf{x}}}\right)=\int_{\mathbb{R}^{2 L} M}\left[\frac{1}{2^{D}} \sum_{i=0}^{2^{D}-1} \Upsilon\left(p_{G}, \mathbf{t}_{i}\right)\right]^{2} d \mathbf{a}
$$

and $\Upsilon\left(p_{G}, \mathbf{t}_{i}\right)=\sum_{q=1}^{L M}\left(\left(\partial^{2} p_{G}\left(\mathbf{a}-\mathbf{t}_{i}\right) / \partial\left(\Re a_{q}\right)^{2}\right)+\left(\partial^{2} p_{G}\right.\right.$ $\left.\left.\left(\mathbf{a}-\mathbf{t}_{i}\right) / \partial\left(\Im a_{q}\right)^{2}\right)\right)$. From (7) the optimal smoothing parameter is found to be

$$
\sigma_{0, \mathrm{opt}}=\left(\left[T \Gamma\left(p_{\hat{\mathbf{x}}}\right)\right]^{-1} 2 L M \int_{\mathbb{R}^{2 L M}} K_{1}^{2}(\mathbf{a}) d \mathbf{a}\right)^{(1 / 2 L M+4)}
$$

with $\int_{\mathbb{R}^{2 L M}} K_{1}^{2}(\mathbf{a}) d \mathbf{a}=(4 \pi)^{-L M} \cdot \Gamma\left(p_{\hat{\mathbf{x}}}\right)$ can be upper bounded by using Cauchy's inequality as it was done in [6] for the univariate case. After upper bounding we obtain that $\Gamma\left(p_{\hat{\mathbf{x}}}\right) \leq \Gamma\left(p_{G}\right)$, where $\Gamma\left(p_{G}\right)=\int_{\mathbb{R}^{2 L} M}\left[\Upsilon\left(p_{G}, \mathbf{0}_{L M}\right)\right]^{2} d \mathbf{a}$. After some calculations it can be shown that

$$
\Gamma\left(p_{G}\right)=L M(L M+1)(4 \pi)^{-L M} \sigma^{-2 L M-4}
$$

yielding a lower bound on $\sigma_{0, \mathrm{opt}}$

$$
\sigma_{0, \mathrm{opt}} \geq \sigma(0.5 T(L M+1))^{-(1 / 2 L M+4)}=\gamma(L M) .
$$

Note that the result shown in [6] is a special case of (11) for $L M=1$. The estimate $\hat{\sigma}_{0}=k_{0} \gamma(L M)$ of $\sigma_{0, \text { opt }}$ gives an asymptotically unbiased and consistent PDF estimator for $k_{0} \in \mathbb{R}$ chosen to be $k_{0} \geq 1$, since $\lim _{T \rightarrow \infty} \hat{\sigma}_{0}=0$ and $\lim _{T \rightarrow \infty} T \hat{\sigma}_{0}=\infty$. For the univariate case, this was shown in [6]. The simulation results given in Fig. 2 show a small variation of the optimal value of $k_{0}$ with respect to $L$ and $N_{I}$, which proves that performance dependence on $D$ is small. Moreover, $k_{0} \approx 2$ is a good choice to be used in practice. Note that no knowledge of $\mathbf{t}_{i}$ or $\mathbf{H}_{I}$ is needed for $\hat{\sigma}_{0 \text {,opt }}$ calculation. We assume that the PDF in (5) remains the same for second and subsequent iterations, which results in same optimal value of $k_{0}$ for all iterations.

Since (4) contains sums of exponentials it can be implemented using the Jacobian algorithm [7]. The complexity per symbol of the proposed method is $O(T L M)$. The conventional $\mathrm{SC} / \mathrm{MMSE}$ receiver's complexity is $O\left(L^{3} M^{3}\right)$.

\footnotetext{
${ }^{1}$ That method would have exponential complexity increase with $D$.
} 


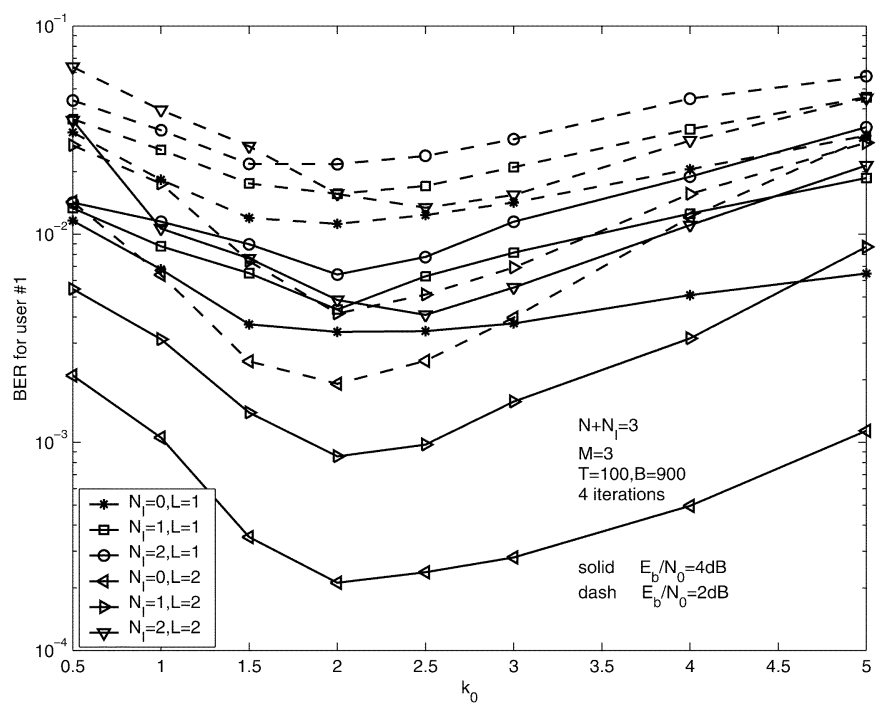

Fig. 2. BER versus $k_{0}$ performance.

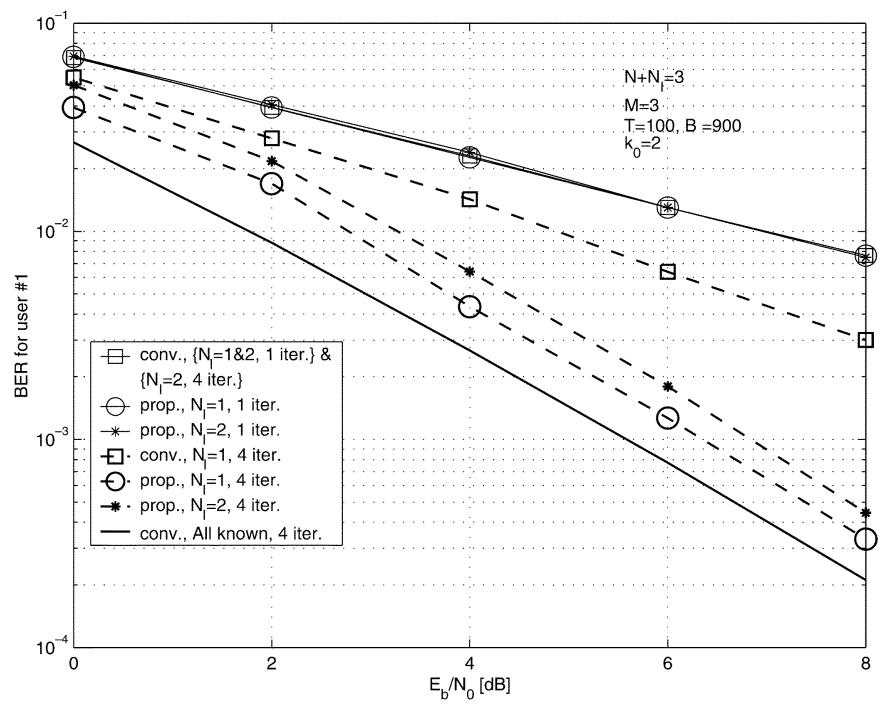

Fig. 3. BER versus $E_{b} / N_{0}$ performance, frequency flat fading.

\section{NUMERICAL EXAMPLES}

The performance of the proposed receiver was tested through simulations. Training sequence length $T=100$, and data sequence length $B=900$. It was assumed that fading is equal path Rayleigh, constant over each frame transmitted, and it changes independently frame by frame. The $R=1 / 2$ convolutional code with generator polynomial $(5,7)_{8}$ and LogMAP decoder [1] were used for all MIMO users. The user specific random interleavers were assumed. Least squares (LS) channel estimation (see [4]) was used.

In Figs. 3 and 4, BER versus per-antenna $E_{b} / N_{0}$ is presented for $L=1$ and $L=2$ cases, respectively. In both cases, the proposed receiver significantly outperforms the conventional one in the case that one or two out of three users are UCCI. This is the consequence of the linear processing of the conventional receiver of [2], that does not take into account the actual structure of the UCCI-plus-noise. Performance curve when all the users

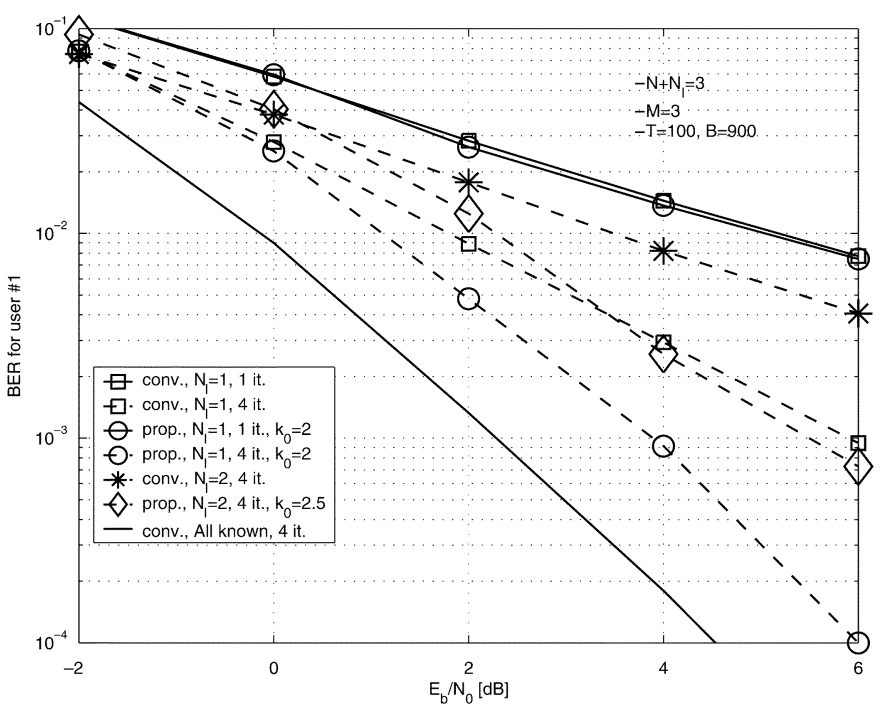

Fig. 4. BER versus $E_{b} / N_{0}$ performance, 2-path fading.

are known and detected is shown for comparison (indicated by "all known"). The performance is closer to the "all known" case for $L=1$ than for $L=2$ and for $N_{I}=1$ than $N_{I}=2$. This is because the PDF of (5) becomes more scattered in the $L M$ dimensional space with increased $L$ and $N_{I}$. It means that fewer samples $\hat{\mathbf{x}}$ (out of $T$ available) effectively contribute to the estimate $\hat{p}_{\hat{\mathbf{x}}}(\mathbf{a})$ of $p_{\hat{\mathbf{x}}}(\mathbf{a})$ in (6), which decreases PDF estimation accuracy. The increased $M$ for fixed $T$ also contributes to the estimation inaccuracy due to the increased dimensionality of $\hat{\mathbf{x}}$ [5]. Its impact is, however, partly compensated by (11).

\section{CONCLUSIONS}

A PDF estimation-based receiver was proposed to preserve the diversity gain of iterative SC/MMSE receivers for MIMO multiuser detection in frequency selective channels in the presence of UCCI. It has been verified through simulations that the proposed receiver significantly outperforms the conventional covariance estimation based method in channels with low memory length, where the degradation is more severe due to the lack of multipath diversity.

\section{REFERENCES}

[1] T. Abe and T. Matsumoto, "Space-time turbo equalization in frequency-selective MIMO channels," IEEE Trans. Veh. Technol., vol. 52, pp. 469-475, May 2003.

[2] T. Abe, S. Tomisato, and T. Matsumoto, "A MIMO turbo equalizer for frequency-selective channels with unknown interference," IEEE Trans. Veh. Technol., vol. 52, pp. 476-482, May 2003.

[3] B. Lu and X. Wang, "Iterative receivers for multiuser space-time coding systems," IEEE J. Select. Areas Commun., vol. 18, pp. 2322-2335, Nov. 2000.

[4] M. Loncar, R. Muller, J. Wehinger, and T. Abe, "Iterative joint detection, decoding and channel estimation for dual antenna arrays in frequency selective fading," in Proc. IEEE Int. Symp. Wireless Personal Mobile Commun., vol. 1, Honolulu, HI, Oct. 27-30, 2002, pp. 125-129.

[5] B. W. Silverman, Density Estimation for Statistics and Data Analysis. New York: Chapman \& Hall, 1986.

[6] C. Luschi and B. Mulgrew, "Nonparametric trellis equalization in the presence of non-Gaussian interference," IEEE Trans. Commun., vol. 51, pp. 229-239, Feb. 2003.

[7] B. Vucetic and J. Yuan, Turbo Codes: Principles and Applications. London, U.K.: Kluwer Academic, 2000. 